\title{
O IMPACTO DA PANDEMIA DE COVID 19 NO DIAGNÓSTICO DE CÂNCER EM UM SERVIÇO DE PATOLOGIA DO SUL DO BRASIL
}

\section{ARTIGO ORIGINAL}

KLOCK, Julia Cristhina Monteiro¹, Borges, Giuliano Santos², OGATA, Daniel Cury ${ }^{3}$, KLOCK, Clóvis ${ }^{4}$

KLOCK, Julia Cristhina Monteiro. Et al. O impacto da pandemia de covid 19 no diagnóstico de câncer em um serviço de patologia do sul do Brasil. Revista Científica Multidisciplinar Núcleo do Conhecimento. Ano. 06, Ed. 11, Vol. 14, pp. 182 -190. Novembro de 2021. ISSN: 2448-0959, Link de acesso: https://www.nucleodoconhecimento.com.br/saude/servico-de-patologia, DOI: 10.32749/nucleodoconhecimento.com.br/saude/servico-de-patologia

\section{RESUMO}

A pandemia do Covid-19 trouxe impacto significativo na vida das pessoas. Um desses impactos pode ser verificado no diagnóstico de outras doenças, em especial, o câncer. Problema: Qual foi o impacto no diagnóstico de novos casos de câncer na região de abrangência de um serviço de patologia do sul do Brasil durante a pandemia? Objetivo geral: Avaliar se houve diminuição no número de diagnósticos de câncer num serviço de Patologia da região sul do Brasil durante a pandemia. Metodologia: Foram analisados os laudos de casos realizados nos Laboratórios do Grupo Infolaudo, durante os meses iniciais da pandemia (abril e maio de 2020) e comparados com os mesmos meses de 2019, a fim de medir qual foi o impacto da pandemia no diagnóstico de câncer em um Serviço de Patologia no Sul do Brasil. Resultados: Comparando os períodos de abril e maio de 2019 com o mesmo

\footnotetext{
${ }^{1}$ Estudante de Medicina - Universidade de Mogi das Cruzes. ORCID: 0000-0002-0834-193X.

${ }^{2}$ Especialidade em Oncologia, Graduação em Medicina. ORCID: 0000-0002-0737-7922.

${ }^{3}$ Doutor em Cirurgia, Mestrado em Cirurgia, Especialidade em Patologia, Graduação em Medicina. ORCID 00000001-8819-2155.

${ }^{4}$ Doutorando em Oncologia, Especialidade em Patologia, Graduação em Medicina. Orcid 0000-0001-84560061.
}

RC: 102325

Disponível em: https://www.nucleodoconhecimento.com.br/saude/servico-depatologia 
período em 2020, percebeu-se a diminuição de $34,2 \%$ nos procedimentos anatomopatológicos. Alguns tipos de câncer como: cólon, esôfago, mama e próstata, tiveram diminuição significativa nos diagnósticos, sendo respectivamente de 19,7\%, 45\%, 18,2\% e 37,7\%. Conclusão: Com o fechamento de muitos consultórios e clínicas nas fases iniciais da pandemia, houve uma diminuição nos diagnósticos de câncer nos laboratórios pesquisados.

Palavras-Chaves: Pandemia, Covid 19, câncer, patologia.

\section{INTRODUÇÃO}

A Organização Mundial da Saúde (OMS) estima que, no ano 2030, haverá 27 milhões de novos casos de câncer, 17 milhões de mortes pela doença e 75 milhões de pessoas vivendo com câncer ao redor do mundo (INCA, 2020).

O câncer é a principal causa de morte nos países desenvolvidos e em desenvolvimento (ALLGAR e NEAL, 2005). No entanto, certos tipos de câncer têm uma alta chance de cura se forem detectados em estágio inicial e tratados adequadamente (AMBUSAIDI e AL-BALUSHI, 2012).

Os atrasos no diagnóstico do câncer podem ocorrer ao longo do diagnóstico, conforme os níveis de atenção à saúde: paciente, atenção primária e atenção secundária. Diagnósticos em doenças neoplásicas mais avançadas podem ocorrer quando há morosidade do paciente em reconhecer e agir em relação aos sintomas suspeitos (ASCO, 2020). Essa baixa conscientização quanto aos sintomas precoces do câncer, é considerada a razão predominante para uma apresentação tardia, principalmente quando os sintomas são de natureza atípica (ASCO, 2020). Outra possível barreira, pode estar relacionada à alta demanda dos serviços médicos especializados, podendo, eventualmente, retardar o diagnóstico, principalmente, nos serviços públicos (INCA, 2020).

No Brasil, segundo o INCA, no ano de 2020, foram estimados 309.230 novos casos de câncer, em homens, o câncer de próstata foi responsável por $29,2 \%$ dos casos 
estimados. Em mulheres, dos 316.140 novos casos esperados, o câncer de mama correspondeu a 29,7\%. Esses dados não incluem o câncer de pele não melanoma (GREEN et al., 2015).

Em suma, o atraso no diagnóstico do câncer pode ocorrer em vários níveis. $O$ paciente pode deixar de reconhecer sintomas suspeitos de câncer ou agir sobre eles. O médico de cuidados primários pode não reconhecer pacientes com suspeitos sintomas de câncer e investigá-los adequadamente ou encaminhá-los a tempo. Pacientes com suspeita clínica, em cuidados secundários, podem não ser atendidos a tempo ou podem ser encaminhados para a especialidade errada.

Durante a pandemia do coronavírus, as medidas preventivas adotadas, como: o distanciamento social e a quarentena, afetaram o diagnóstico inicial de pacientes com câncer. Assim como, a superlotação observada em diversos hospitais, impossibilitou o adequado diagnóstico e tratamento destes pacientes.

Neste cenário, faltam dados da casuística local, para verificar se a pandemia afetou mesmo esse grupo de pacientes e os diagnósticos de câncer.

Diante disto, o presente artigo tem como questão norteadora: Qual foi o impacto no diagnóstico de novos casos de câncer na região de abrangência de um serviço de patologia do sul do Brasil durante a pandemia? Tendo como objetivo geral avaliar se houve diminuição no número de diagnósticos de câncer num serviço de patologia da região sul do Brasil durante a pandemia

Foram analisados os dados do início da pandemia (abril e maio) de 2020, comparando com os dados de abril e maio de 2019, de um Serviço de Patologia da Região Sul do Brasil (Grupo Infolaudo) para o reconhecimento da situação atual e os impactos resultantes do período de lockdown no diagnóstico deste grupo de indivíduos.

Trata-se de um estudo epidemiológico transversal e quantitativo, da casuística local. 


\section{MATERIAIS E MÉTODOS}

Foram analisados todos os laudos de casos do período de abril e maio de 2020 e abril e maio de 2019, realizados nos Laboratórios de Grupo Infolaudo, sendo os mesmos comparados entre si. Os períodos escolhidos foram definidos devido ao início das medidas de isolamento social e lockdown. Todos os pacientes incluídos neste estudo foram atendidos na região da Foz do rio Itajaí, Alto vale, grande Florianópolis e região norte e oeste do Rio Grande do Sul (Erechim e Cruz Alta).

Todos os casos com diagnóstico de câncer foram incluídos no estudo. Foram excluídos os casos que não possuíam confirmação do diagnóstico de câncer. Os dados coletados foram incorporados ao programa Microsoft Office Excel® 2010 e os resultados foram apresentados em números absolutos e porcentagens. Trata-se de um estudo epidemiológico transversal e quantitativo da casuística local.

\section{DISCUSSÃO}

Atrasos na atenção primária podem ocorrer no reconhecimento, investigação e encaminhamento de casos com suspeita de neoplasia maligna (ASCO, 2020). Embora a atenção primária seja o primeiro ponto de contato dos pacientes que utilizam os serviços nacionais de saúde em vários países do mundo, o atraso no diagnóstico do câncer permanece como um problema contínuo nesse nível (JONES et al., 2007). Espera-se que os médicos da atenção primária identifiquem os pacientes com possível doença em um estágio inicial. Entretanto, o diagnóstico de câncer é relativamente incomum para o médico da atenção primária, pois mais de $80 \%$ dos pacientes apresentam sintomas inespecíficos (HARFORD, 2011). De fato, a principal preocupação destes profissionais da área da saúde, é diferenciar a minoria dos pacientes que precisam de atenção urgente, daqueles que provavelmente têm condições autolimitadas (KAUFMAN et al., 2020).

Em maio de 2020, a Sociedade Americana de Oncologia Clínica (ASCO) publicou um relatório especial recomendando o adiamento de quaisquer visitas a clínicas e 
qualquer rastreamento de câncer ou diagnóstico e procedimentos relacionados ao estadiamento, se este adiamento não representar um risco de progressão da doença ou agravamento do prognóstico (LONDON et al., 2020).

Alguns trabalhos internacionais mostram que a diminuição do diagnóstico de câncer nos primeiros meses da pandemia foi de $65,2 \%$ de novos casos de câncer (LONDON et al., 2020).

O screening de alguns tipos de câncer foi prejudicado, com dados mostrando que o câncer de mama e de cólon e reto foram os mais afetados com 89,2 e 84,5\% respectivamente (MACLEOD et al., 2009).

Em um estudo realizado no Reino Unido, o lockdown ocasionou a suspensão das triagens oncológicas, comprometendo o diagnóstico precoce de inúmeros pacientes. Apenas disso, os pacientes com quadro clínico crítico e sintomático foram direcionados à intervenção diagnóstica. Utilizou-se registros de câncer no National Health Service (NHS) por meio de banco de dados de hospitais com pacientes de 15 a 84 anos, com diagnóstico de câncer de mama (35583), colorretal (24975) e esofágico (6744) em 2010 com acompanhamento até 2014. Nos pacientes com neoplasia primária de pulmão (29305), foi utilizado 2012 como ano de diagnóstico e 2015 como data final de seguimento. Por meio de um fluxograma para definir os caminhos dos pacientes oncológicos dentro do NHS, foi realizada uma estimativa para avaliar as consequências do atraso diagnóstico neste grupo de pacientes, durante um período de 12 meses, começando em março de 2020 (data do lockdown), contextualizando com o seu impacto 1, 3 e 5 anos após o diagnóstico inicial. Nesta metodologia, foram consideradas três vias ou fluxos destes pacientes, correspondendo do melhor para o pior cenário. Com base nisso, estimou-se o impacto real de sobrevida em 1, 3 e 5 anos após o diagnóstico, calculando assim, o total de óbitos atribuídos por câncer e o total de anos de vida perdidos, comparando com dados pré-pandêmicos. 
Em todos os cenários, estimou-se um acréscimo de 7,9\% a 9,6\% de mortes por câncer de mama em até 5 anos após o diagnóstico, significando de 281 a 344 mortes a mais, respectivamente. No câncer colorretal, o aumento foi de 15,3\% (1445) a 16,6\% (1563) e nos cânceres de pulmão, esse aumento foi de 4,8\% (1235) a 5,3\% (1372). E finalmente, o aumento observado nos pacientes com câncer de esôfago foi de 5,8\% (330) a 6\% (342). Estes dados mostram que houve um aumento significativo de mortes evitáveis no Reino Unido, provavelmente devido às medidas restritivas e de isolamento social (MARINGE et al., 2020).

Outro estudo publicado por Kaufman et al. (2020), observou redução de $40 \%$ na incidência semanal de câncer na Holanda e 75\% no Reino Unido desde o início da pandemia de covid-19. Este estudo utilizou uma metodologia semelhante à nossa, avaliando os registros de um banco de dados de janeiro a abril de 2019, comparando com o mesmo período de 2020. Notaram quedas significativas nos diagnósticos de neoplasias malignas, in situ e de comportamento indeterminado. Durante a pandemia, o número semanal de casos caiu mais de $46 \%$ nos seis tipos mais comuns de câncer, com variações de $24,7 \%$ nos casos de neoplasia do pâncreas e $51,8 \%$ nos casos de mama (WHO, 2016).

Outro artigo, publicado por pesquisadores do Sidney Kimmel Center, da Filadélfia e do NHS, evidenciaram uma redução de $89,2 \%$ no rastreamento dos cânceres da mama e $85,5 \%$ de diminuição das colonoscopias, que é uma importante ferramenta para o diagnóstico precoce do câncer colorretal (VOSE, 2020).

Todos os casos do Grupo Infolaudo nos períodos de março e abril de 2020 foram comparados com o período de março e abril de 2019. Os meses de março e abril foram selecionados, pois foram os meses iniciais de lockdown.

No ano de 2019, nos meses de abril e maio os Laboratórios do Grupo Infolaudo receberam 5524 procedimentos anatomopatológicos, destes 4634 foram lesões benignas e 890 lesões malignas, representando $16,1 \%$. No ano de 2020 , no mesmo período, foram 3635 procedimentos, com 2875 lesões benignas e 760 de lesões 
malignas, representando as malignas $20,9 \%$. Houve uma diminuição de $34,2 \%$ de procedimentos anatomopatológicos. (Gráfico 1)

Gráfico 1: Diminuição dos procedimentos anatomopatológicos nos meses de abril e maio de 2020 em relação ao mesmo período de 2019.

\section{Lesões benignas X Lesões malignas}

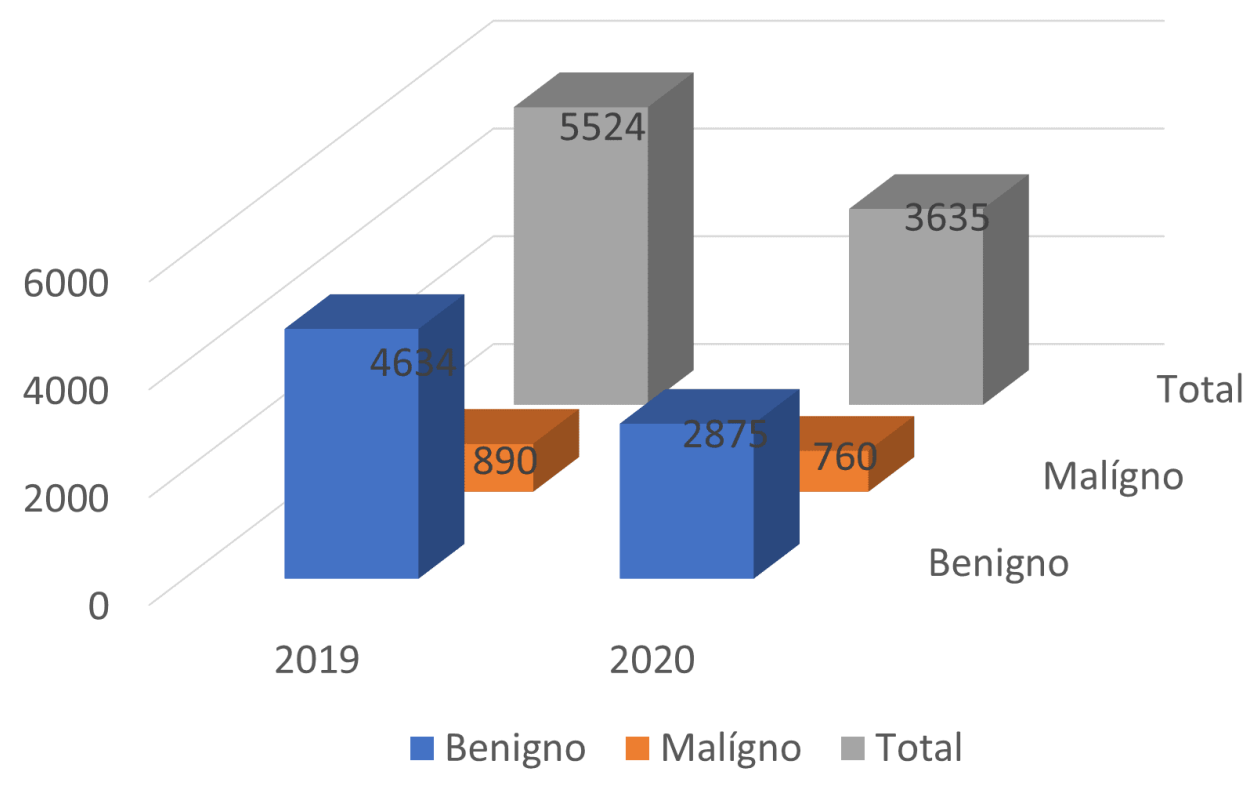

Fonte: autores

No geral, em todos os procedimentos diferentes, houve uma diminuição no diagnóstico, sejam as biópsias ou as peças cirúrgicas. Alguns tipos de procedimentos tiveram uma diminuição mais acentuada, como as grandes cirurgias eletivas que necessitavam de internação do paciente.

Alguns tipos de câncer (Gráfico 2), tiveram uma diminuição mais significativa, como de cólon (19,7\%), esôfago (45\%), mama (18,2\%) e próstata $(37,7 \%)$.

RC: 102325

Disponível em: https://www.nucleodoconhecimento.com.br/saude/servico-depatologia 
Gráfico 2: diminuição dos diagnósticos de câncer (por órgãos) mais afetados nos meses de abril e maio de 2020 em relação ao mesmo período de 2019.

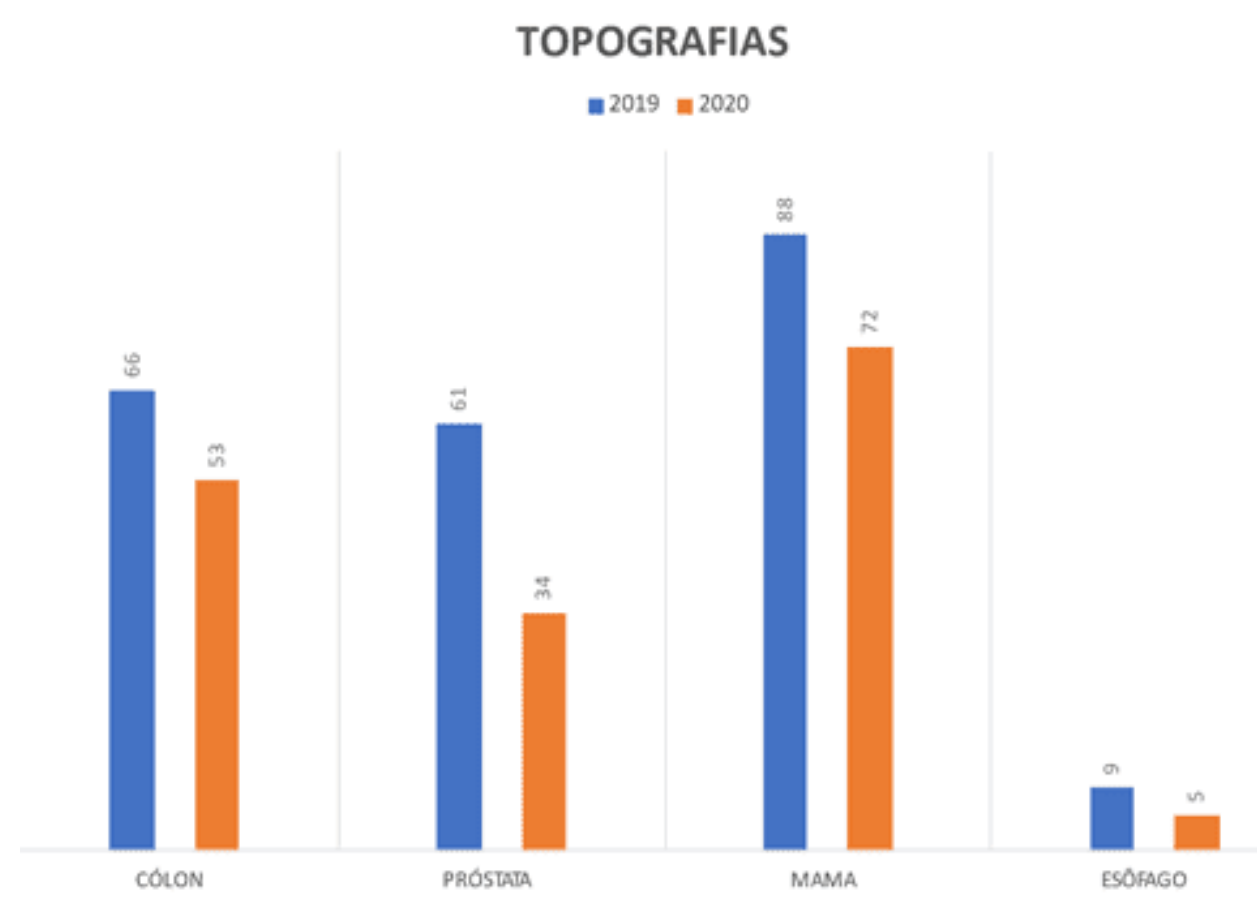

Fonte: autores.

A principal diminuição foi de casos que necessitariam de algum procedimento invasivo, e em casos de sedação do paciente. Outros tipos, como os cânceres de pele, não tiveram diminuição no mesmo período.

Os blocos cirúrgicos de grande parte dos hospitais também limitaram as cirurgias eletivas, em virtude da necessidade de deixar vagos os leitos, especialmente os leitos de UTIs.

Com o impacto do fechamento de muitos consultórios e clínicas nas fases iniciais da pandemia, houve um impacto grande nesses diagnósticos. Além do medo dos pacientes em sair de suas casas, o que também foi um dos fatores que ajudou nessa diminuição. 
Este estudo tem algumas limitações. Em primeiro lugar, foram avaliados apenas o impacto do bloqueio no diagnóstico em um grupo de laboratórios, embora seja um serviço grande. Os resultados do presente estudo, portanto, não são necessariamente aplicáveis a outros centros, sendo assim, estudos multicêntricos seriam necessários para validar esses resultados em escala nacional. É possível que isso tenha induzido um viés de seleção. No entanto, este método de seleção de pacientes foi idêntico para todos os períodos estudados, permitindo comparabilidade.

\section{CONSIDERAÇÕES FINAIS}

Retomando a questão norteadora: Qual foi o impacto no diagnóstico de novos casos de câncer na região de abrangência de um serviço de patologia do sul do Brasil durante a pandemia? Por meio do estudo apresentado, foi possível concluir que os dados analisados nos laudos dos pacientes atendidos no Grupo Infolaudo, mostraram que o diagnóstico de alguns tipos de câncer teve uma diminuição no período inicial da pandemia no ano de 2020 com o período analisado de 2019, mostrando que a pandemia afetou esse tipo de diagnóstico. Também houve uma diminuição grande no número de biópsias e de peças cirúrgicas recebidas no período. Essa explicação pode ser multifatorial, podendo ser influenciada pelo fechamento dos serviços diagnósticos como clínicas e setores dos hospitais, bem como pelo medo dos pacientes em procurarem atendimento médico.

\section{REFERÊNCIAS}

ALLGAR, V. L.; NEAL, R. D. Delays in the diagnosis of six cancers: analysis of data from the National Survey of NHS Patients: Cancer. Br J Cancer, 2005.

AMBUSAIDI, A.; AL-BALUSHI, S. Educação em Saúde no Sultanato de Omã. In: Taylor, N. et al. (ed.). Educação em Saúde em Contexto. Editores Sense. 2012. 
ASCO. American Society of Clinical Oncology. COVID-19 Patient Care Information. 2020. Disponível em: https://www.asco.org/asco-coronavirus-information/careindividuals-cancer-during-covid-19. Acesso em: 26 de jun. de 2020.

GREEN, T. et al. Cancer detection in primary care: insights from general practitioners. Ir. J Cancer, 2015.

HARFORD, J. B. Breast-cancer early detection in low-income and middleincome countries: do what you can versus one size fits all. Lancet Oncol, 2011.

INCA. Instituto Nacional de Câncer. Estimativa 2020: incidência de câncer no Brasil. Rio de Janeiro: INCA, 2020.

JONES, R. et al. Alarm symptoms in early diagnosis of cancer in primary care: cohort study using General Practice Research Database. BMJ, 2007.

KAUfMAN, H. W. et al. Changes in the Number of US Patients With Newly Identified Cancer Before and During the Coronavirus Disease 2019 (COVID-19) Pandemic. JAMA Netw Open, 2020.

LONDON, J. W. et al. Effects of the COVID-19 pandemic on cancer-related patient encounters. JCO Clinical Cancer Inform, 2020.

MACLEOD, $U$. et al. Risk factors for delayed presentation and referral of symptomatic cancer: evidence for common cancers. Br J Cancer, 2009.

MARINGE, C. et al. The impact of the COVID-19 pandemic on cancer deaths due to delays in diagnosis in England, UK: a national, population-based, modelling study. Lancet Oncol, 2020.

VOSE, J. M. Delay in Cancer Screening and Diagnosis During the COVID-19 Pandemic: What Is the Cost? Oncology (Williston Park), 2020. 
WHO. World Health Organization. Facts about cancer. 2016. Disponível em: www.who.int

Enviado: Novembro de 2021.

Aprovado: Novembro de 2021. 